PROCEEDINGS OF THE

AMERICAN MATHEMATICAL SOCIETY

Volume 49, Number 2, June 1975

\title{
CONES OF POTENTIALS
}

\author{
BRUCE CALVERT
}

ABSTRACT. We construct a cone of potentials from a $T$-monotone operator and use this to give smoothness of supersolutions solving a minimum problem.

Introduction. In the different theories of potential there are cones of "superharmonic" functions. In considering the essential properties of such cones, Mokobodzki has defined a cone of potentials, emphasising the reduction of a function. This paper relates this notion to the nonlinear potential theory of the author. The property (2) of cones of potentials is then exploited to give regularity of solutions to variational problems, under the hypotheses of Lewy and Stampacchia [10].

Notation. Let $(E, \leq)$ be an ordered vector space, $E^{+}$the positive cone of $E$, and $C \subset E^{+}$a convex cone. One says following Mokobodzki [13], that $(C, \leq)$ defines a cone of potentials if the following conditions are satisfied:

(1) For all $u$ and $v$ in $C$, the set $M(u-v)=\{w$ in $C: w \geq u-v\}$ possesses a minimum for the order $\leq$ called $R(u-v)$.

(2) One has $(u-R(u-v)) \in C$ for all $u, v \in C$.

Let $(W, \leq)$ be a vector lattice which is a Banach space, such that the positive cone is closed. Let $V$ be a closed reflexive subspace which also is a sublattice, with dual $V^{*}$. One says that the operator $A$ from $W$ to $P\left(V^{*}\right)$, the set of subsets of $V^{*}$, is monotone if for $u$ and $v$ in $W$, such that $u-v$ is in $V$, and $u^{*}$ in $A u$ and $v^{*}$ in $A v,\left(u^{*}-v^{*}, u-v\right) \geq 0$. A is maximal monotone means it is not properly contained in another monotone operator. One says $A: W \rightarrow P\left(V^{*}\right)$ is $T$-monotone if for $u^{*}$ in $A u, v^{*}$ in $A v$, and $(u-v)^{+}$ in $V,\left(u^{*}-v^{*},(u-v)^{+}\right) \geq 0$. Some examples appear in [1], [2] and [5]. One says $A$ is strictly $T$-monotone if equality for some $u^{*}$ and $v^{*}$ implies $(u-v)^{+}=$ 0 . By the domain $D(B)$ of $B: V \rightarrow P\left(V^{*}\right)$ we mean $x$ such that $B x$ is non-

Received by the editors September 10, 1973.

AMS (MOS) subject classifications (1970). Primary 47H05; Secondary $31 \mathrm{~B} 35$, $31 \mathrm{C} 25,35 \mathrm{~J} 60,47 \mathrm{~A} 20$.

Key words and phrases. T-monotone, principle of the lower envelope, cone of potentials, Hölder continuous. 
empty. We say $B: V \rightarrow P\left(V^{*}\right)$ is coercive to mean $(B u, u) /\|u\| \rightarrow \infty$ as $\|u\| \rightarrow \infty$, and $B^{-1}$ is locally bounded if every $f \in V^{*}$ has a neighbourhood $N$ such that $\{x \in V: B x \cap N$ is nonempty $\}$ is bounded. For $K$ a closed convex nonempty subset of $V, \partial \psi_{K}: V \rightarrow P\left(V^{*}\right)$ is defined by $u^{*} \in \partial \psi_{K} u$ if $u \in K$ and $\forall v \in K,\left(u^{*}, u-v\right) \geq 0$. By a generalised duality map $J$, one means that there is a prescribed strictly increasing continuous function $\psi$ from $\{r \in R$ : $r \geq 0\}$ to itself, $\psi(0)=0, \psi(r) \rightarrow \infty$ as $r \rightarrow \infty$, and $J: V \rightarrow P\left(V^{*}\right)$ is defined by $\left\|v^{*}\right\|=\psi\|v\|$ and $\left(v^{*}, v\right)=\left\|v^{*}\right\| \cdot\|v\|$ for $v^{*}$ in $J v . V$ is a Banach lattice means $\sup (x,-x) \leq \sup (y,-y)$ implies $\|x\| \leq\|y\|$. We say the norm is monotonic if $0 \leq x \leq y$ implies $\|x\| \leq\|y\|$. The cone is normal means there is an equivalent monotonic norm.

Theorem 1. Let $V$ be a vector lattice, which is also a reflexive Banach space, with closed positive cone $H$. Suppose $V$ and $V^{*}$ are strictly convex. Suppose B: $V \rightarrow V^{*}$ is maximal monotone, and $d \in D(B)$. Suppose:

(a) A generalised duality map $J$ preserves order, $V$ is a Banach lattice, and $B$ is strictly $T$-monotone; or

(b) A generalised duality map $J$ is strictly $T$-monotone, the norm in $V$ is monotonic, and $B$ is T-monotone.

Then $B+\partial \psi_{d+H}$ is maximal monotone, as is $B+\partial \psi_{d-H}$.

Proof. We use the following result from Calvert [6].

Lemma. Let $X$ be a reflexive Banach space, $X$ and $X^{*}$ strictly convex. $S$ uppose $J$ is a generalised duality map and $K$ a closed nonempty convex subset of $X$. Let $A: X \rightarrow P\left(X^{*}\right)$ be maximal monotone. Suppose that for all $\lambda>0,\left(I+\lambda J^{-1} A\right)^{-1} K \subset K$. Then $A+\partial \psi_{K}$ is maximal monotone.

By replacing $B$ in the statement of Theorem 1 by $x \rightarrow B(x+d)-d^{*}$, where $d^{*} \in B(d)$, we may assume $d=0$ and $0 \in B(0)$. By the lemma we have to show that if $u+\lambda J^{-1} u^{*}=f \geq 0, u^{*} \in B(u), \lambda>0$, then $u \geq 0$. We need the following result from Calvert [7].

Lemma. Suppose $X$ is an ordered Banach space with $X^{*}$ strictly convex, and monotonic norm. Then $x \geq 0, y \geq 0$ implies $(J x, y) \geq 0$. If $X$ is also a Banach lattice then $x \perp y$ implies $(J x, y)=0$.

Assuming (a), since $J$ is order preserving,

$$
\left(0-u^{*},(0-\dot{u})^{+}\right)=\left(J \lambda^{-1}(u-f),(-u)^{+}\right) \leq\left(J \lambda^{-1}\left(u^{+}\right),(-u)^{+}\right)=0
$$

by the lemma. Since $B$ is strictly $T$-monotone, $u \geq 0$. Assuming (b), the $T$ - 
monotonicity of $B$ gives $\left(J(f-u),(-u)^{+}\right) \leq 0$. Since $f \geq 0, J f \geq 0$ by the lemma. It follows that $\left(J(f-u)-J f,((f-u)-f)^{+}\right) \leq 0$. Since $J$ is strictly $T$-monotone, $u \geq 0$. Q.E.D.

Theorem 1 gives conditions under which the maximality assumptions of Theorem 2 are fulfilled. The maximality of $B+\partial \psi_{d+H}$ in Theorem 1 holds also under

(c) interior $D(B) \cap(H+d) \neq \varnothing$.

This follows from Rockafellar [15], and where $B$ is a function from $V$ to $V^{*}$, the result follows from Hartman and Stampacchia [8], and Browder [3]. The properties (1) and (2) of Theorem 2 are the same as those of Mokobodzki, for if $A: W \rightarrow V^{*}$ is a bounded linear operator then $C=\{x \geq 0$ in $W: A x \geq 0\}$ is a convex cone, and by linearity, if $u, v \in C$ then $A(u-v) \leq A u$. We take $f=0$. By linearity, $(u-R(u-v)) \in C$ is the same as $A R(u-v) \leq A u$.

Theorem 2. Let $V$ and $W$ be as above. Let $H$ be the cone in $V$. Suppose $A: W \rightarrow P\left(V^{*}\right)$ is strictly $T$-monotone, and for $c$ in $D(A)$ the two operators from $V$ to $P\left(V^{*}\right)$,

$$
u \rightarrow A(u+c)+\partial \psi_{H}, \quad u \rightarrow A(u+c)+\partial \psi_{-H},
$$

are maximal monotone. Suppose $u \rightarrow A(u+c)$ is coercive. Then $\{u \in W$ : $\left.u \geq 0, \exists u^{*} \in A u, u^{*} \geq 0\right\}$ satisfies the conditions (1) and (2) of a cone of potentials:

(1) Suppose $u, v \in W, f \in V^{*}$, and $\sup (f, A(u-v), A(0)) \leq A u$, i.e. $\exists(u-v)^{*} \in A(u-v), u^{*} \in A u, 0^{*} \in A(0)$ such that $\sup \left(f,(u-v)^{*}, 0^{*}\right) \leq u^{*}$. Then $\left\{w \geq(u-v)^{+}: \exists w^{*} \in A w, w^{*} \geq f\right\}$ has a minimum element $R(u-v)$.

(2) $\exists(R(u-v))^{*} \in A R(u-v)$ such that $(R(u-v))^{*} \leq u^{*}$, for every $u^{*} \in A u$ as in (1), i.e. such that $\exists(u-v)^{*} \in A(u-v)$, and $\exists 0^{*} \in A(0)$ satisfying $\sup \left(f,(u-v)^{*}, 0^{*}\right) \leq u^{*}$.

Proof. From Rockafellar [16] we use

Theorem. Let $X$ be a reflexive Banach space, and let $B: X \rightarrow P\left(X^{*}\right)$ be maximal monotone. Then $B$ is surjective if and only if $B^{-1}$ is locally bounded.

The coercivity of $u \rightarrow A(u+c)$ gives coercivity of the two operators concerned and, hence, their surjectivity.

Proof of (1). This is essentially contained in Lions and Stampacchia [11, Theorem 6.2], as well as Lewy and Stampacchia [9, Lemma 1.1].

By the theorem above there is $u_{0}$ in $H$ and $u_{0}^{*}$ in $A\left(u_{0}+(u-v)^{+}\right)$such 
that for all $w$ in $H,\left(u_{0}^{*}-f, u_{0}-w\right) \leq 0$. We set $R(u-v)=u_{0}+(u-v)^{+}$. Then for $w$ in $(u-v)^{+}+H$,

$$
\left(u_{0}^{*}-f, R(u-v)-w\right) \leq 0 .
$$

This implies $u_{0}^{*} \geq f$.

Suppose $w \geq(u-v)^{+}$and there is $w^{*}$ in $A w, w^{*} \geq f$.

$$
\begin{aligned}
\left(u_{0}^{*}-w^{*},(R(u-v)-w)^{+}\right) & \leq\left(u_{0}^{*}-f,(R(u-v)-w)^{+}\right) \\
& =\left(u_{0}^{*}-f, R(u-v)-\inf (w, R(u-v))\right) \leq 0 .
\end{aligned}
$$

Since $A$ is strictly $T$-monotone, $R(u-v) \leq w$.

Proof of (2). This is indicated by Lewy and Stampacchia [9] in their remark after Theorem 3.1, proved for the classical case by Lewy and Stampacchia [10, Theorem 2.1], and in a general potential theory by Mokobodzki [12].

By the theorem above, there exists $x$ in $R(u-v)-H$ and $x^{*}$ in $A x$ such that for all $y$ in $R(u-v)-H$,

$$
\left(x^{*}-u^{*}, x-y\right) \leq 0 .
$$

Since $(u-v)^{*} \leq u^{*}$, and $\sup ((u-v), x) \leq R(u-v)$,

$$
\left((u-v)^{*}-x^{*},(u-v-x)^{+}\right) \leq\left(u^{*}-x^{*}, \sup (u-v, x)-x\right) \leq 0 .
$$

By the strict monotonicity of $A, x \geq u-v$. Since $0^{*} \leq u^{*}$, and $x^{+} \leq R(u-v)$,

$$
\left(0^{*}-x^{*},(0-x)^{+}\right) \leq\left(u^{*}-x^{*}, x^{+}-x\right) \leq 0 .
$$

By the strict monotonicity of $A, x \geq 0$. Hence $x \geq(u-v)^{+}$and we may put $x=w$ in (3) to give $\left(u_{0}^{*}-f, R(u-v)-x\right) \leq 0$. By putting $R(u-v)=y$ in (4) we obtain $\left(x^{*}-u^{*}, x-R(u-v)\right) \leq 0$. Since $u^{*} \geq f,\left(f-u^{*}, R(u-v)-x\right) \leq$ 0 . Adding these three inequalities gives

$$
\left(u_{0}^{*}-x^{*}, R(u-v)-x\right) \leq 0 .
$$

Since $A$ is strictly monotone, $R(u-v)=x$, and since $x^{*} \leq u^{*}$ by (4), we have shown that (2) holds. Q.E.D.

Corollary 1. Suppose $V, W$, and $A$ are as in Theorem 2, except $A$ is Tmonotone instead of strictly $T$-monotone. Suppose that there is a reflexive Banach space $U$ which is a vector lattice with closed cone containing $W$ as a dense subspace and as a sublattice, and that the cone in $U$ is normal. Suppose there exists $F: U \rightarrow U^{*}$, strictly T-monotone, demicontinuous and bounded, and $c \geq 0$ implies $F(c) \geq 0$. Then the conclusion of Theorem 2 bolds if $f$ and $A u$ are in $U^{*}$. 
Proof. For $n$ a positive integer, $A+n^{-1} F$ satisfies the conditions of Theorem 2, where we regard $F$ as an operator from $W$ to $V^{*}$. Therefore there is $w_{n}$, and $w_{n}^{*}$ in $A w_{n}$, such that

$$
w_{n}^{*}+\partial \psi_{(u-v)}+{ }_{+H} w_{n}+n^{-1} F w_{n} \ni f .
$$

By coercivity, $\left\{w_{n}: n \in Z^{+}\right\}$is bounded in $W$. Since $F$ is bounded, $n^{-1} F w_{n}$. $\rightarrow 0$ in $U^{*}$. Suppose a subsequence $w_{n^{\prime}}-(u-v)^{+}$converges weakly in $V$

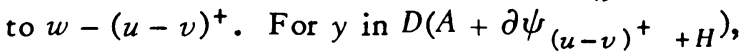

$$
\left(\left(A+\partial \psi_{(u-v)}^{+}+{ }_{+}\right) y-f+n^{-1} F w_{n}, y-w_{n}\right) \geq 0
$$

where we mean that inequality holds for all elements of $\left(A+\partial \psi_{(u-v)^{+}+H^{+}}\right) y$. Taking limits,

$$
\left(\left(A+\partial \psi_{(u-v)}+{ }_{+H}\right) y-f, y-w\right) \geq 0,
$$

which gives $f \in\left(A+\partial \psi(u-v)^{+}+{ }_{+}\right) w$.

Suppose $w_{0} \geq(u-v)^{+}, w_{0}^{*}$ in $A w_{0}, w_{0}^{*} \geq f$. Since $n^{-1} F\left(w_{0}\right) \geq 0, w_{0}^{*}+$ $n^{-1} F w_{0} \geq f$. Consequently, $w_{0} \geq w_{n}$. Taking the weak limit for the subsequence $n^{\prime}$ gives $w_{0} \geq w$. This proves (1) of Theorem 2. We show (2) holds. We note that for $m>n$,

$$
w_{m}^{*}+n^{-1} F w_{m} \geq w_{m}^{*}+m^{-1} F w_{m} \geq f
$$

giving $w_{m} \geq w_{n}$, so that $\left(w_{n^{\prime}}\right)$, being an increasing sequence converging weakly to $w$ in $U$, is also strongly convergent by normality of the cone. Since $\left\{w_{n}^{*}\right\}$ is order bounded in $U^{*}$, it is bounded and, hence, a subsequence of $w_{n^{\prime}}^{*}$ converges weakly in $U^{*}$ to $w^{*}$, say. Since $A$ restricted to $\left\{w: A w \subset U^{*}\right\}$ is maximal monotone as an operator from $U$ to $P\left(U^{*}\right), w^{*}$ is in $A w$. Taking limits in $w_{n}^{*}+n^{-1} F w_{n} \leq u^{*}+n^{-1} F u$ gives $w^{*} \leq u^{*}$. Q.E.D.

Supposing $A$ is monotone, a converse to Theorem 2 would give $T$ monotonicity if $\{u \geq 0: A u \geq 0\}$ were a "cone of potentials". The next four results show when $\{u \geq 0: A u \geq 0\}$ being a cone of potentials is equivalent to the principle of the lower envelope and when the principle of the lower envelope is equivalent to $T$-monotonicity. We say $A: W \rightarrow V^{*}$ satisfies the principle of the lower envelope if $A \inf (x, y) \geq \inf (A x, A y)$ and the principle of the upper envelope if $A \sup (x, y) \leq \sup (A x, A y)$.

Proposition 1. Suppose $V$ and $W$ are as above, and $A: W \rightarrow V^{*}$ satisfies the principles of the upper and lower envelopes. Suppose that for $c$ in $W$, 
$u \rightarrow A(c+u)$ is strictly monotone, demicontinuous, and coercive from $V$ to $V^{*}$. Then the conclusion of Theorem 2 holds .

Proof. One follows the proof of Theorem 2, using the principle of the upper envelope to prove (1) and the principle of the lower envelope to prove (2).

Proposition 2. Suppose $V$ and $W$ are as above, and $A: W \rightarrow V^{*}$ is given. Suppose that for $u$ and $v$ in $W$ and $f$ in $V^{*}$ the set $\{w \geq u-v: A w \geq f\}$ has a minimum. Then A satisfies the principle of the lower envelope.

Proof. Given $x$ and $y$ in $W$, set $u-v=\inf (x, y)$ and $f=\inf (A x, A y)$.

Proposition 3. Suppose $V$ and $W$ are as above, and $A: W \rightarrow V^{*}$ satisfies the principle of the lower envelope. Suppose $k>0$ and for $x-y$ in $V$, $(A x-A y, x-y) \geq k\|x-y\|^{2}$. Suppose $u \rightarrow A(c+u)$ is bounded and demicontinuous from $V$ to $V^{*}$ for $c$ in $W$. Then $A$ is T-monotone.

Proof. By [4, Proposition 1.4], $A$ satisfies the domination principle. By [4, Proposition 1.5], if $e>0,(u-v)^{+} \in V$ and $(I+e A) u \leq(I+e A) v$, then $u \leq v$. By the proof of [4, Proposition 1.1], the modulus contraction acts with respect to $A$. By [4, Proposition 1.2], $A$ is $T$-monotone.

Proposition 4. Suppose $V$ and $W$ are as above, and there exists $F: W \rightarrow$ $V^{*}$, strictly $T$-monotone and demicontinuous. Suppose $A: W \rightarrow V^{*}$ is $T$ monotone, and for $c$ in $W, u \rightarrow A(u+c)$ is demicontinuous and coercive from $V$ to $V^{*}$. Then $A$ satisfies the principles of the upper and lower envelopes.

Proof. One follows the proof of [4, Proposition 1.3], using T-monotonicity instead of assuming that the modulus contraction operates.

Theorem 3. Let $M$ be a compact n-dimensional Riemannian manifold with nonempty boundary. Let $m \in(1, \infty)$. Let $W^{1, m}$ be the closure of the Lipschitz functions $u: M \rightarrow R$ in the norm $\left(\int|\operatorname{grad} u|^{m}+|u|^{m}\right)^{1 / m}$, integration being with respect to the Riemannian measure. Define $A: W^{1 / m} \rightarrow W^{-1, m^{\prime}}$ by

$$
A u=-\operatorname{div}\left(|\operatorname{grad}(u)|^{m-2} \operatorname{grad}(u)\right) .
$$

Suppose $\psi \in W^{1, m}, \psi \leq 0$ on bdy $(M), \psi \in L^{\infty}$, and the positive Radon measure $(A \psi)^{+}$on $M-\operatorname{bdy}(M)$ satisfies, for all closed balls $B(x, r)$,

$$
(A \psi)^{+} B(x, r) \leq C r^{n-m+a}, \quad 0<\alpha<1 .
$$

Then $R(\psi)$, the minimal element of $\left\{w \geq \psi^{+}: A w \geq 0\right\}$ exists and is Hölder continuous in $M-b d y(M)$. 
Remark. This result does not give as high an exponent, in the case $m=2$, as was obtained by Lewy and Stampacchia [10, Theorem 3.2]. We note that other regularity results follow from property (2) of a cone of potentials; if $(A \psi)^{+} \in L^{p}$ (or $W^{-1, p}$ ), then $A R(\psi) \in L^{p}$ (or $W^{-1, p}$ ).

Proof. From Morrey [14] we recall

Theorem 3.52. Suppose $u \in W^{1, m}\left[B\left(x_{0}, R\right)\right], 1 \leq m \leq n$, and for $x$ in $B\left(x_{0}, R\right), 0 \leq r \leq \delta=R-\left|x-x_{0}\right|$,

$$
\int_{B(x, r)}|\operatorname{grad} u|^{m} d x \leq L^{m}(r / \delta)^{n-m+m \mu} .
$$

Then $u \in C_{\mu}^{0}\left[B\left(x_{0}, r\right)\right]$ for each $r<R$ and

$$
|u(y)-u(x)| \leq C L \delta^{1-n / m}(|y-x| / \delta)^{\mu}
$$

for $|y-x| \leq \delta / 2, C=C(n, m, \mu)$.

Since $A$ is strictly $T$-monotone, demicontinuous and coercive, $R(\psi)$ exists and by (2) of Theorem 2 satisfies $A R(\psi) B(x, r) \leq C r^{n-m+a}$. We assume $m \leq n$, for otherwise $u$ is Hölder continuous anyway. Take $\alpha^{\prime}=$ $\alpha m /(n+\alpha)$ and $\gamma=1-\alpha /(n+\alpha)$. We see that $n-\gamma m=n-m+\alpha^{\prime}$ and $(n-m+\alpha) y=n-m+\alpha^{\prime}$. We denote all constants independent of $r$ and $u$ by $C$. Given $r$ small, we take $\zeta, 0 \leq \zeta \leq 1, \zeta=1$ on $B(x, r)$, with support in $B\left(x, r^{\gamma}\right) \subset M-\operatorname{bdy}(M),|\operatorname{grad} \zeta| \leq C r^{-\gamma}$.

By $(1), R(\psi) \leq\left\|\psi^{+}\right\|_{L^{\infty}}$ We may take continuous functions, $\leq\left\|\psi^{+}\right\|_{L^{\infty}}$, with support in $B\left(x, r^{\gamma}\right)$, convergent to $\zeta^{m} R(\psi)$ in $W^{1, m}$, giving

$$
\left(A R(\psi), \zeta^{m} R(\psi)\right) \leq C r^{\gamma(n-m+\alpha)}=C r^{n-m+a^{\prime}} .
$$

This implies that

$$
\int|\operatorname{grad} R(\psi)|^{m} \zeta^{m} \leq C r^{n-m+a^{\prime}}+C \int R(\psi) \zeta^{m-1}|\operatorname{grad} \zeta||\operatorname{grad} R(\psi)|^{m-1} .
$$

By Young's inequality

$$
\begin{aligned}
\int|\operatorname{grad} R(\psi)|^{m} \zeta^{m} & \leq C r^{n-m+\alpha^{\prime}}+C \int|R(\psi)|^{m}|\operatorname{grad} \zeta|^{m} \\
& \leq C r^{n-m+\alpha^{\prime}}+C r^{n-\gamma m}=C r^{n-m+a^{\prime}} .
\end{aligned}
$$

Hence

$$
\int_{B(x, r)}|\operatorname{grad} R(\psi)|^{m} \leq C r^{n-m+a^{\prime}} .
$$

By Morrey's theorem, $R(\psi)$ is Hölder continuous of exponent $\alpha^{\prime} / m=$ $\alpha /(n+\alpha)$. Q.E.D. 
We now give an application of Corollary 1 of Theorem 2. Suppose $M$ as above, and for $x$ almost everywhere in $M, a_{x}: T_{x}^{*} \rightarrow T_{x}$ is a monotone continuous map from the cotangent to the tangent space at $x$, satisfying $\left(a_{x}(p)\right.$, $p) \geq C_{1}|p|^{m},\left|a_{x}(p)\right| \leq C_{2}|p|^{m-1}$, and such that in local coordinates the map $x \rightarrow a_{x}(p)$ is measurable for $p$ in $R^{n}$, giving a map $a: T^{*}(M) \rightarrow T(M)$.

Define $A u=-\operatorname{div} a(d u)$.

Corollary 2. Let $M, W^{1, m}, 1 \leq m \leq n$, be as in Theorem 3, $A$ as above, $\psi$ in $W^{1, m} \cap L^{\infty}, \psi \leq 0$ on bdy $M,(A \bar{\psi})^{+} \in L^{p}, 1<p^{-1}<m n^{-1}$, and $p^{-1} \leq$ $1-m^{-1}+n^{-1}$ if $m<n$. Then $R(\psi)$ exists and is Hölder continuous on $M-\operatorname{bdy}(M)$, with constant $(p m-n) /(p n+p m-n)$.

Proof. Since $p<\infty$, and $1-p^{-1} \geq m^{-1}-n^{-1}$ if $m<n, W^{1, m} \subset L^{p^{\prime}}$, and we apply Corollary 1 of Theorem 2 with $U=L^{p^{\prime}}$. As $F$ we may take the duality map for $L^{p^{\prime}}$. This gives, if we set $\alpha=m-n p^{-1}>0$

$$
A R(\psi) B(x, r) \leq C\left\|A(\psi)^{+}\right\|_{L^{p}} r^{n / p^{\prime}} \leq C r^{n-m+a} .
$$

By the proof of Theorem 3, $R(\psi)$ is Hölder continuous with exponent $\alpha /(n+\alpha)=(p m-n) /(p n+p m-n)$. Q.E.D.

\section{REFERENCES}

1. H. Brézis and G. Stampacchia, Sur la régularité de la solution d'inéquations elliptiques, Bull. Soc. Math. France 96 (1968), 153-180. MR 39 \#659.

2. H. Brézis, Problèmes unilatéraux, J. Math. Pures Appl. 51 (1972), 1-168.

3. F. Browder, Nonlinear monotone operators and convex sets in Banach spaces, Bull. Amer. Math. Soc. 71 (1965), 780-785. MR 31 \#5112.

4. B. Calvert, Potential theoretic properties for monotone operators, Boll. Un. Mat. Ital. (4) 5 (1972), 473-489. MR 46 \#9812.

314. MR 47 \#5677.

5. - On T-accretive operators, Ann. Mat. Pura Appl. (4) 94 (1972), 291 -

6. - Maximal monotonicity and m-accretivity of $A+B$, Atti Accad. Naz. Lincei Rend. Cl. Sci. Fis. Mat. Natur. (8) 49 (1970), (1971), 357-363. MR 46 \#2501.

7. - Nonlinear equations of evolution, Pacific J. Math. 39 (1971), 293350. MR $47 \# 7531$.

8. P. Hartman and G. Stampacchia, On some non-linear elliptic differentialfunctional equations, Acta Math. 115 (1966), 271-310. MR 34 \#6355.

9. H. Lewy and G. Stampacchia, On the regularity of the solution of a variational inequality, Comm. Pure Appl. Math. 22 (1969), 153-188. MR 40 \#816.

10. - On the smoothness of superharmonics which solve a minimum problem, J. Analyse Math. 23 (1970), 227-236. MR 42 \#6266.

11. J. Lions and G. Stampacchia, Variational inequalitites, Comm. Pure Appl. Math. 20 (1967), 493-519. MR 35 \#7178.

12. G. Mokobodzki, Cônes de potentiels et noyaux subordonnés, Potential Theory (C.I.M.E., I Ciclo, Stresa, 1969), Edizioni Cremonese, Rome, 1970, pp. 207248. MR 43 \#551. 
13. G. Mokobodzki, Structure des cones de potentiels, Séminaire Bourbaki, 22é année, 1969/70, no. 377, Lecture Notes in Math., vol. 180, Springer-Verlag, Berlin, 1971, pp. 239-252.

14. C. Morrey, Multiple integrals in the calculus of variations, Die Grundlehren der math. Wissenschaften, Band 130, Springer-Verlag, New York, 1966. MR 34 \#2380.

15. R. Rockafellar, On the maximality of sums of nonlinear monotone operators, Trans. Amer. Math Soc. 149 (1970), 75-88. MR 43 \#7984.

16. - Local boundedness of nonlinear, monotone operators, Michigan Math. J. 16 (1969), 297-407. MR 40 \#6229.

DE PAR TMENT OF MATHEMATICS, UNIVERSITY OF AUCKLAND, AUCKLAND, NEW ZEALAND 